\title{
Deployment of titanium thermal barrier for low-temperature carbon nanotube growth
}

\author{
G. Y. Chen, ${ }^{\text {a) }}$ C. H. P. Poa, S. J. Henley, V. Stolojan, and S. R. P. Silva \\ Nano-Electronic Centre, Advanced Technology Institute, School of Electronics and Physical Science, \\ University of Surrey, Guildford, Surrey GU2 7XH, United Kingdom \\ Sajad Haq \\ Advanced Technology Centre, Sowerby Building, BAE SYSTEMS, Filton FPC 267, Bristol BS34 7QW, \\ United Kingdom
}

(Received 23 May 2005; accepted 9 November 2005; published online 16 December 2005)

\begin{abstract}
Chemical vapor-synthesized carbon nanotubes are typically grown at temperatures around $600{ }^{\circ} \mathrm{C}$. We report on the deployment of a titanium layer to help elevate the constraints on the substrate temperature during plasma-assisted growth. The growth is possible through the lowering of the hydrocarbon content used in the deposition, with the only source of heat provided by the plasma. The nanotubes synthesized have a small diameter distribution, which deviates from the usual trend that the diameter is determined by the thickness of the catalyst film. Simple thermodynamic simulations also show that the quantity of heat, that can be distributed, is determined by the thickness of the titanium layer. Despite the lower synthesis temperature, it is shown that this technique allows for high growth rates as well as better quality nanotubes. (c) 2005 American Institute of Physics. [DOI: 10.1063/1.2150587]
\end{abstract}

Carbon nanotubes are potentially useful materials for a host of applications, such as electrodes, ${ }^{1,2}$ field emission devices, ${ }^{3,4}$ hydrogen storage, ${ }^{5-7}$ etc. This is in part due to their nanometer sizes and high aspect ratios. The formation of this material by self-assembly alleviates the need for expensive lithography. However, the temperature at which it is normally synthesized restricts its application to certain areas, such as deployment on flexible substrates, or its compatibility with subsequent processes (e.g., unavoidable drive-in diffusion of dopants when the intended application is as interconnects in silicon hybrid devices).

Recently, there have been reports of low-temperature synthesis of carbon nanotubes. ${ }^{8,9}$ However, low-temperature synthesis typically results in amorphous carbon or carbon nanofibers, rather than nanotubes. The formation of carbon nanotubes is typically carried out at temperatures above $900{ }^{\circ} \mathrm{C}$. In the conventional growth mechanism however, the role of the substrate plays no part, other than as support. This means that there is only a requirement for the catalyst to be sufficiently heated and not the substrate. A simple way of achieving this is to use a layer with lower thermal conductivity to restrict the transfer of heat to the substrate. It is shown in this letter that a combination of a titanium layer and reduced hydrocarbon concentration can be used to achieve nanotube growth at low substrate temperatures, using plasma-enhanced chemical vapor deposition (CVD).

A parallel-plate direct current-PECVD system is used in the growth, with acetylene as the carbon feedstock and nitrogen and hydrogen as the carrier/dilution gas. The system base pressure is $10^{-3}$ Torr and the graphite substrate table temperature is monitored using an embedded thermocouple. The temperature reading is taken as the highest temperature reached during deposition. The concentration of hydrocarbon was simply taken as the gas flow ratio of acetylene to a nitrogen and hydrogen mixture. An electrode separation of

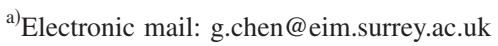

$1 \mathrm{~cm}$ was used in all depositions. $30 \mathrm{~nm}$ nickel films were deposited on $500 \mathrm{~nm}$ of titanium with silicon (100) substrate and were not pretreated in any form before the carbon nano-
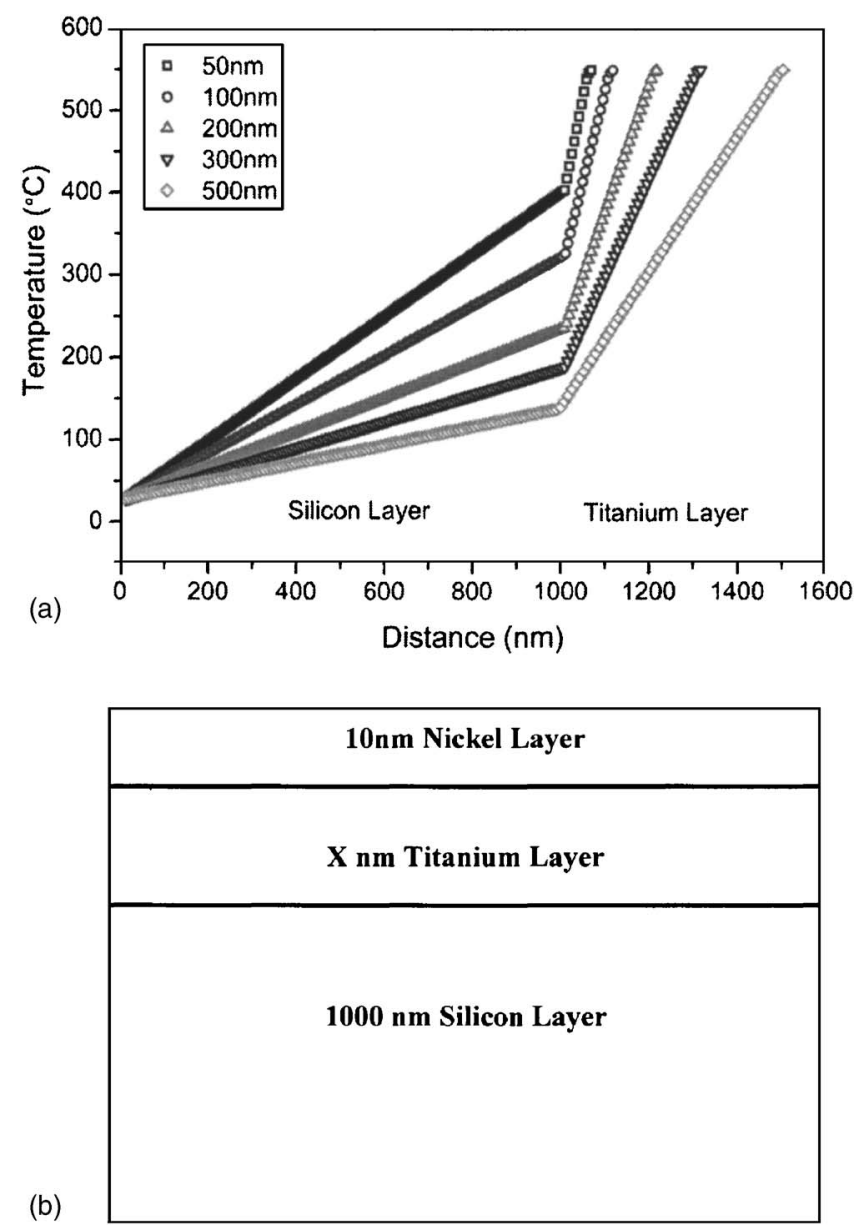

FIG. 1. (a) Thermodynamic simulation of the effect of different titanium layer and (b) layout of simulated structure. 

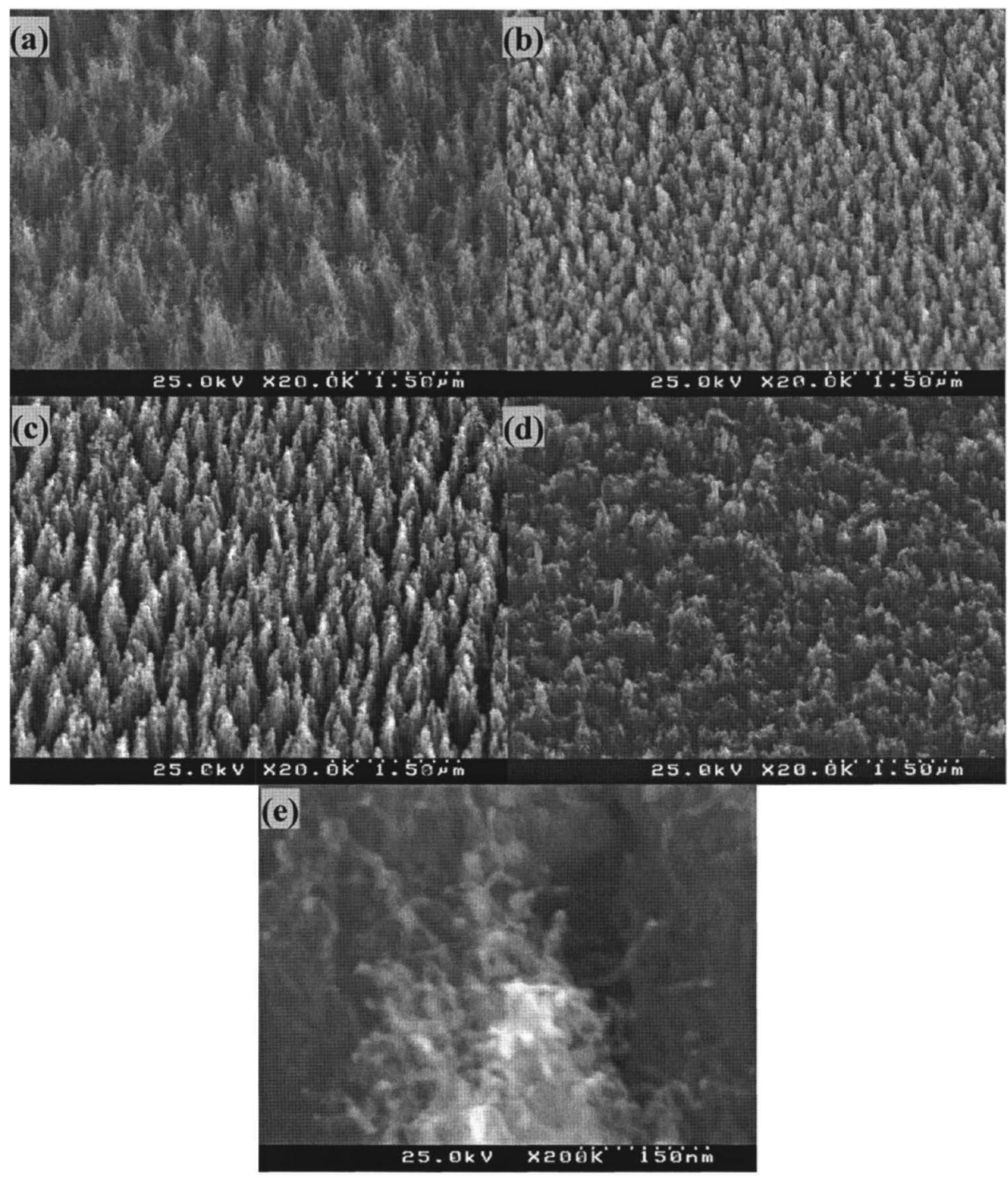

FIG. 2. SEM image of the effect of different hydrocarbon concentrations: (a) $1 \%$, (b) $2 \%$, (c) $3 \%$, (d) $6 \%$, and (e) higher magnification image of (a). tube deposition. A typical run would start with the backfilling of the evacuated chamber to the desired operating pressure with the nitrogen and hydrogen mixture, and then igniting the plasma before the introduction of acetylene. The deposition was carried out for $45 \mathrm{~min}$. After deposition, the sample was allowed to cool to below $50{ }^{\circ} \mathrm{C}$ in a nitrogen ambient, before sample removal. The empirical observation of the morphology and microstructure of the nanotubes were conducted using a Hitachi S-4000 field emission scanning electron microscope (SEM) and Philips CM200 high-resolution transmission electron microscope (HRTEM). The HRTEM is operated at $200 \mathrm{kV}$ with a $\mathrm{LaB}_{6}$ filament and has a line resolution of $0.14 \mathrm{~nm}$.

The low thermal conductivity of titanium (among the metals) means it can be utilised as an effective thermal barrier. A simple thermodynamic simulation shows that, at steady state, large thermal gradients can be achieved using increasing layer thicknesses [Fig. 1(a)]. The structure simulated is based on a double layer consisting of $10 \mathrm{~nm}$ nickel on different thicknesses of titanium, on a $1000 \mathrm{~nm}$ silicon substrate [Fig. 1(b)]. In order to simulate the concept of surface plasma heating and the thermal barrier, the surface is kept at $550{ }^{\circ} \mathrm{C}$ while the base of the substrate is kept at $27^{\circ} \mathrm{C}$. The important point here is that the titanium layer can be used to hold significant plasma heating on the surface Downloaded 30 Mar 2009 to 131.227.178.132. Redistribution subje while allowing the substrate to be maintained at a much lower temperature, purely due to its lower thermal conductivity.

Carbon nanotubes were synthesized with the plasma ignited at $-500 \mathrm{~V}$ bias at 10.3 Torr. The growth was carried out for $45 \mathrm{~min}$. The effect of varying the hydrocarbon concentration on the growth is shown in Fig. 2.

The maximum temperature reached was $392{ }^{\circ} \mathrm{C}$, with the lowest temperature only reaching $371^{\circ} \mathrm{C}$ [Fig. 2(a)]. The temperature stabilized within $10 \mathrm{~min}$, suggesting that equilibrium had been reached. In a typical CVD process without any deliberate modifications, nanotube growth would not be observed at these temperatures. For all hydrocarbon variations, nanotube growth has been observed, with the quantity of amorphous carbon increasing as the hydrocarbon concentration increases. With $1 \%$ hydrocarbon concentration, the synthesized nanotubes appear as tightly packed vertically aligned bundles. Once the concentration is increased, the growth resembles clumps of amorphous carbon, with decreasing nanotube yield. Upon reaching 6\%, the film begins to form into amorphous carbon thin films. It is important to note that, without the titanium layer, no growth was observed at such low temperatures. There were no observable changes in the diameters of the synthesized carbon nanotubes, as the hydrocarbon concentration was varied. The nanotubes' outer to AIP license or copyright; see http://apl.aip.org/apl/copyright.jsp 


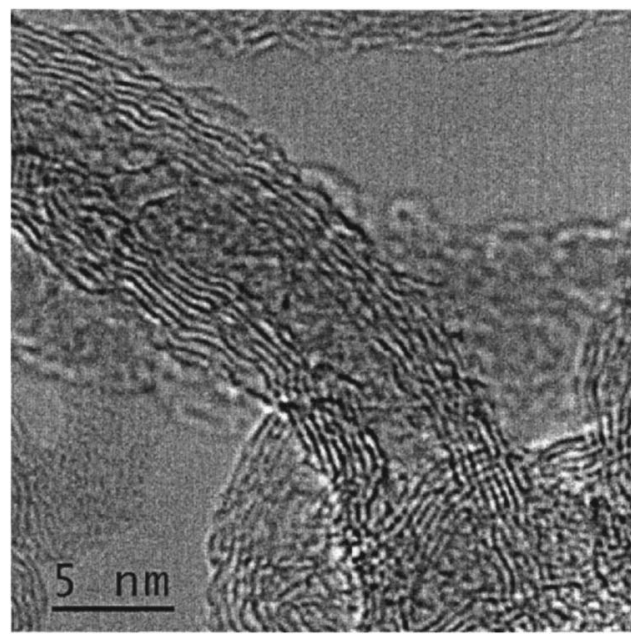

FIG. 3. HRTEM of low-temperature synthesized carbon nanotube with inner diameter of $1.6 \mathrm{~nm}$ and outer diameter of $7 \mathrm{~nm}$.

diameters are typically around 5-12 nm, with the number of graphene layers ranging from 8 to over 20 layers. This is unusual, since the diameters of the nanotubes are typically dependent on the thickness of the catalyst films, i.e., nanotube diameters are either similar or larger than the films' thickness. ${ }^{10}$ This frequently observed trend is due to the nanotube nucleating from the catalyst nanoparticles. The morphology of the carbon nanotubes is analyzed using HRTEM, as shown in Fig. 3.

The sample was obtained by scratching the surface with tweezers and subsequent ultrasonication in methanol. The solution was then filtered through a holey carbon grid. The inner core of the nanotube can be clearly seen, with the graphene planes oriented parallel to the nanotube axis. This technique, of using the barrier layer and plasma heating, is demonstrated to be able to synthesize carbon nanotubes. Conventional lower temperature processes typically yield carbon nanofibers. ${ }^{8,9,11}$ The approach of surface heating and substrate cooling will allow for the synthesis of the carbon nanotubes at low substrate temperatures. It is also possible that graphitization can be improved, with increased surface heating.

The technique shown here utilises both the low thermal conductivity of titanium and the low concentration of hydrocarbon. In plasma-assisted growth, the surface heating from the plasma is often ignored, or assumed to be similar to the set substrate temperature. The significance of plasma heating has been highlighted by Teo et al., ${ }^{12}$ where it was reported that plasma heating using $200 \mathrm{~W}$ can result in the substrate heating up to $700{ }^{\circ} \mathrm{C}$. However, this is a general statement which we do not apply to many growth chambers, as the heating depends on the plasma power density $\left(20 \mathrm{~W} \mathrm{~cm}^{2}\right.$ in Ref. 12). For our experiment, the power density was only $5.37 \mathrm{~W} \mathrm{~cm}^{2}$, which is only about one-quarter of the power shown to result in a substrate heating of $700{ }^{\circ} \mathrm{C}$. The titanium layer not only allows significant heat to be held close to the surface, it also provides a conductive layer below the nanotubes. With suitable cooling, this would ensure that the substrate is kept at low temperatures, considerably below $700{ }^{\circ} \mathrm{C}$. The flux of carbon arriving at the catalyst surface plays a crucial role. Growth at low substrate temperatures does not allow for rapid diffusion and for the interaction of carbon in/with the nickel catalyst. Therefore, if a typical hydrocarbon concentration of $5 \%$ or greater is used, this rapidly leads to thin-filmlike formation which inhibits the nucleation and formation of nanostructures.

In conclusion, carbon nanotubes can be synthesized at relatively low substrate temperatures, using a combination of a titanium barrier layer and low hydrocarbon concentrations, in a plasma-assisted growth technique. The use of titanium as a thermal barrier will allow the possibility of maintaining the substrate temperature close to room temperature, while allowing the growth of carbon nanotubes with high levels of graphitization.

${ }^{1}$ C. Niu, E. K. Sichel, R. Hoch, D. Moy, and H. Tennet, Appl. Phys. Lett. 70, 1480 (1997).

${ }^{2}$ Y. Tzeng, Y. Chen, and C. Liu, Diamond Relat. Mater. 12, 774 (2003).

${ }^{3}$ Z. Sun, Y. J. Liu, G. Y. Chen, S. P. Lau, B. K. Tay, J. S. Chen, and L. K. Cheah, Surf. Rev. Lett. 18, 505 (2001).

${ }^{4}$ W. J. Yu, Y. S. Cho, G. S. Choi, D. J. Kim, Y. H. Song, and J. H. Lee, Diamond Relat. Mater. 13, 1017 (2004).

${ }_{5}^{5}$ J. Kong, N. R. Franklin, C. Zhou, M. G. Chapline, S. Peng, K. Cho, and H. Dai, Science 287, 622 (2000).

${ }^{6}$ G. G. Tibbetts, G. P. Meisner, and C. H. Olk, Carbon 39, 2291 (2001).

${ }^{7}$ Y. Ye, C. C. Ahn, C. Witham, B. Fultz, J. Liu, G. Rinzler, D. Colbert, K. A. Smith, and R. E. Smalley, Appl. Phys. Lett. 74, 2307 (1999).

${ }^{8}$ S. Hofmann, B. Kleinsorge, C. Ducati, and J. Robertson, New J. Phys. 5, 153.1 (2003).

${ }^{9}$ T. M. Minea, S. Point, A. Granier, and M. Touzeau, Appl. Phys. Lett. 85, 1244 (2004).

${ }^{10}$ C. H. P. Poa, S. J. Henley, G. Y. Chen, A. A. D. T. Adikaari, C. E. Giusca, and S. R. P. Silva, J. Appl. Phys. 97, 114308 (2005).

${ }^{11}$ B. O. Boskovic, V. Stolojan, R. U. A. Khan, S. Haq, and S. R. P. Silva, Nat. Mater. 1, 165 (2002).

${ }^{12}$ K. B. K. Teo, D. B. Hash, R. G. Lacerda, N. L. Rupesinghe, M. S. Bell, S. H. Dalal, D. Bose, T. R. Govindan, B. A. Cruden, M. Chhowalla, G. A. J. Amaratunga, M. Myyappan, and W. I. Milne, Nano Lett. 4, 921 (2004). 